\title{
Stellar Evolution in the Early Universe
}

\author{
Raphael Hirschi ${ }^{1,2}$, Urs Frischknecht ${ }^{3}$, F.-K. Thielemann ${ }^{3}$, Marco \\ Pignatari $^{1,6}$, Cristina Chiappini ${ }^{4,5}$, Sylvia Ekström ${ }^{4}$, \\ Georges Meynet ${ }^{4}, \&$ André Maeder ${ }^{4}$ \\ ${ }^{1}$ Astrophysics group, Keele University, Lennard-Jones Lab., Keele, ST5 5BG, UK \\ email: r.hirschi@epsam.keele.ac.uk \\ ${ }^{2}$ IPMU, University of Tokyo, Kashiwa, Chiba 277-8582, Japan \\ ${ }^{3}$ Dept. of Physics \& Astronomy, University of Basel, CH-4056, Basel, Switzerland \\ ${ }^{4}$ Observatoire Astronomique de l'Université de Genève, CH-1290, Sauverny, Switzerland \\ ${ }^{5}$ Osservatorio Astronomico di Trieste, Via G. B. Tiepolo 11, I-34131 Trieste, Italia \\ ${ }^{6}$ JINA, University of Notre Dame, Notre Dame, IN 46556, USA
}

\begin{abstract}
Massive stars played a key role in the early evolution of the Universe. They formed with the first halos and started the re-ionisation. It is therefore very important to understand their evolution. In this paper, we describe the strong impact of rotation induced mixing and mass loss at very low metallicity $(Z)$. The strong mixing leads to a significant production of primary ${ }^{14} \mathrm{~N},{ }^{13} \mathrm{C}$ and ${ }^{22} \mathrm{Ne}$. Mass loss during the red supergiant stage allows the production of Wolf-Rayet stars, type Ib,c supernovae and possibly gamma-ray bursts (GRBs) down to almost $Z=0$ for stars more massive than $60 M_{\odot}$. Galactic chemical evolution models calculated with models of rotating stars better reproduce the early evolution of $\mathrm{N} / \mathrm{O}, \mathrm{C} / \mathrm{O}$ and ${ }^{12} \mathrm{C} /{ }^{13} \mathrm{C}$. We calculated the weak s-process production induced by the primary ${ }^{22} \mathrm{Ne}$ and obtain overproduction factors (relative to the initial composition, $Z=10^{-6}$ ) between 100-1000 in the mass range 60-90 $M_{\odot}$.
\end{abstract}

Keywords. Stars: mass loss, stars: Population II, stars: rotation, stars: supernovae, stars: WolfRayet, Galaxy: evolution

\section{Introduction}

Massive stars $\left(M \gtrsim 10 M_{\odot}\right)$ started forming about 400 millions years after the Big Bang and ended the dark ages by re-ionising the Universe. They therefore played a key role in the early evolution of the Universe and it is important to understand the properties and the evolution of the first stellar generations to determine the feedback they had on the formation of the first cosmic structures. It is unfortunately not possible to observe the first massive stars because they died a long time ago but their chemical signature can be observed in low mass halo stars (called EMP stars), which are so old and metal poor that the interstellar medium out of which these halo stars formed are thought to have been enriched by one or a few generations of massive stars. Since the re-ionisation, massive stars have continuously injected kinetic energy (via various types of supernovae) and newly produced chemical elements (by both hydrostatic and explosive burning and $\mathrm{s}$ and $\mathrm{r}$ processes) into the interstellar medium of their host galaxies. They are thus important players for the chemo-dynamical evolution of galaxies. Most massive stars leave a remnant at their death, either a neutron star or a black hole, which often leads to the formation of pulsars or X-ray binaries.

The evolution of stars is governed by three main parameters, which are the initial mass, metallicity $(Z)$ and rotation rate. The evolution is also influenced by the presence of magnetic fields and of a close binary companion. For massive stars around solar metallicity, mass loss plays a crucial role, in some cases removing more than half of the initial 
mass. Internal mixing, induced mainly by convection and rotation also significantly affect the evolution of stars. The properties of non-rotating low-Z stars are summarised in Sect. 2 of Hirschi et al. (2008). In short, at low Z, stars are more compact, usually have weaker winds (see however Pulstilnik et al. 2008) and are more massive (Bromm \& Loeb 2003, Schneider et al. 2006). The fate of non-rotating massive single stars at low $\mathrm{Z}$ is summarised in Heger et al. (2003) and several groups have calculated the corresponding stellar yields (Heger \& Woosley 2002, Chieffi \& Limongi 2004, Tominaga et al. 2007). In this paper, after discussing the impact of rotation induced mixing and mass loss at low $Z$, we present the implications for the nucleosynthesis and for galactic chemical evolution in the context of extremely metal poor stars.

\section{Rotation, internal mixing and mass loss}

Massive star models including the effects of both mass loss and especially rotation better reproduce many observables around solar $Z$. For example, models with rotation allow chemical surface enrichments already on the main sequence (MS), whereas without the inclusion of rotation, self-enrichments are only possible during the red super-giant (RSG) stage (Heger \& Langer 2000, Meynet \& Maeder 2000). Rotating star models also better reproduce the ratio of WR to $\mathrm{O}$ type stars and also the ratio of type $\mathrm{Ib}+\mathrm{Ic}$ to type II supernovae as a function of metallicity compared to non-rotating models, which underestimate these ratios (see contribution by Georgy in this volume and Meynet \& Maeder 2005). The models at very low $Z$ presented here use the same physical ingredients as the successful solar $Z$ models. The value of $300 \mathrm{~km} \mathrm{~s}^{-1}$ used as the initial rotation velocity at solar metallicity corresponds to an average velocity of about $220 \mathrm{~km} \mathrm{~s}^{-1}$ on the main sequence (MS) which is close to the average observed value. One of the first surveys of OB stars (and in particular their surface velocities) was obtained by Fukuda (1982) and the most recent surveys are listed in Meynet et al. (2008). It is unfortunately not possible to observe very low $Z$ massive stars and measure their rotational velocity since they all died a long time ago. The higher observed ratio of Be to $\mathrm{B}$ stars in the Magellanic clouds compared to our Galaxy (Maeder et al. 1999, Martayan et al. 2007) could point to the fact that stars rotate faster at lower metallicities. Also a low- $Z$ star having the same ratio of surface velocity to critical velocity, $v / v_{\text {crit }}$ (where $v_{\text {crit }}$ is the velocity for which the centrifugal force balances the gravitational force) as a solar- $Z$ star has a higher surface rotation velocity due to its smaller radius (one quarter of $Z_{\odot}$ radius for a very low $Z 20 M_{\odot}$ star). In the models presented below, the initial ratio $v / v_{\text {crit }}$ is the same or slightly higher than for solar Z (see Hirschi 2007 for more details). This corresponds to initial surface velocities in the range of $600-800 \mathrm{~km} \mathrm{~s}^{-1}$. These fast initial rotation velocities are supported by chemical evolution models of Chiappini et al. (2006b) discussed in the next section. The mass loss prescriptions used in the Geneva stellar evolution code are described in detail in Meynet \& Maeder (2005). In particular, the mass loss rates depend on metallicity as $\dot{M} \sim\left(Z / Z_{\odot}\right)^{0.5}$, where $Z$ is the mass fraction of heavy elements at the surface of the star.

How do rotation induced processes vary with metallicity? The surface layers of massive stars usually accelerate due to internal transport of angular momentum from the core to the envelope. Since at low $Z$, stellar winds are weak, this angular momentum dredged up by meridional circulation remains in the star, and the star more easily reaches critical rotation. At the critical limit, matter can easily be launched into a keplerian disk which probably dissipates under the action of the strong radiation pressure of the star.

The efficiency of meridional circulation (dominating the transport of angular momentum) decreases towards lower Z because the Gratton-Öpik term of the vertical velocity 
of the outer cell is proportional to $1 / \rho$. On the other hand, shear mixing (dominating the mixing of chemical elements) is more efficient at low $Z$. Indeed, the star is more compact and therefore the gradients of angular velocity are larger and the mixing timescale (proportional to the square of the radius) is shorter. This leads to stronger internal mixing of chemical elements at low Z (Meynet \& Maeder 2002).

The history of convective zones (in particular the convective zones associated with shell $\mathrm{H}$ burning and core He burning) is strongly affected by rotation induced mixing (see Hirschi 2007). The most important rotation induced mixing takes place while helium is burning inside a convective core. Primary carbon and oxygen are mixed outside of the convective core into the H-burning shell. Once the enrichment is strong enough, the Hburning shell is boosted (the CNO cycle depends strongly on the carbon and oxygen mixing at such low initial metallicities). The shell then becomes convective and leads to an important primary nitrogen production. In response to the shell boost, the core expands and the convective core mass decreases. At the end of He burning, the CO core is less massive than in the non-rotating model. Additional convective and rotational mixing brings the primary CNO to the surface of the star. This has consequences for the stellar yields. The yield of ${ }^{16} \mathrm{O}$, being closely correlated with the mass of the $\mathrm{CO}$ core, is therefore reduced due to the strong mixing. At the same time the carbon yield is slightly increased. The relatively "low" oxygen yields and "high" carbon yields are produced over a large mass range at $Z=10^{-8}$ (Hirschi 2007). This is one possible explanation for the possible high $[\mathrm{C} / \mathrm{O}]$ ratio observed in the most metal-poor halo stars (see Fig. 14 in Spite et al. 2005 and Fabbian et al. 2008) and in DLAs (Pettini et al. 2008).

Models of metal-free stars including the effect of rotation (see contribution by Ekström and Ekström et al. 2008) show that stars may lose up to $10 \%$ of their initial mass due to the star rotating at its critical limit (also called break-up limit). The mass loss due to the star reaching the critical limit is non-negligible but not important enough to drastically change the fate of the metal-free stars. The situation is very different at very low but non-zero metallicity (Meynet et al. 2006, Hirschi 2007). The total mass of an $85 M_{\odot}$ model at $Z=10^{-8}$ is shown in Fig. 1 (left) by the top solid line. This model, like metal-free models, loses around $5 \%$ of its initial mass when its surface reaches breakup velocities in the second part of the MS. At the end of core $\mathrm{H}$ burning, the core contracts and the envelope expands, thus decreasing the surface velocity and its ratio to the critical velocity. The mass loss rate becomes very low again until the star crosses the HR diagram and reaches the RSG stage. In the cooler part of the H-R diagram, the mass loss becomes very important. This is due to the dredge-up by the convective envelope of CNO elements to the surface increasing its overall metallicity. The total metallicity, $Z$, is used in this model (including CNO elements) for the metallicity dependence of the mass loss. Therefore depending on how much CNO is brought up to the surface, the mass loss becomes very large again. The CNO brought to the surface comes from primary $\mathrm{C}$ and $\mathrm{O}$ produced in the He-burning region and from primary $\mathrm{N}$ produced in the H-burning one.

The fate of rotating stars at very low $\mathrm{Z}$ is therefore probably the following:

- $M<40 M_{\odot}$ : mass loss is insignificant and matter is only ejected into the ISM during the SN explosion (see contributions by Nomoto and Tominaga in this volume), which could be very energetic if fast rotation is still present in the core at the core collapse.

- $40 M_{\odot}<M<60 M_{\odot}$ : mass loss (at critical rotation and in the RSG stage) removes $10-20 \%$ of the initial mass of the star. The star probably dies as a black hole without a SN explosion and therefore the feedback into the ISM is only due to stellar winds, which are slow. 

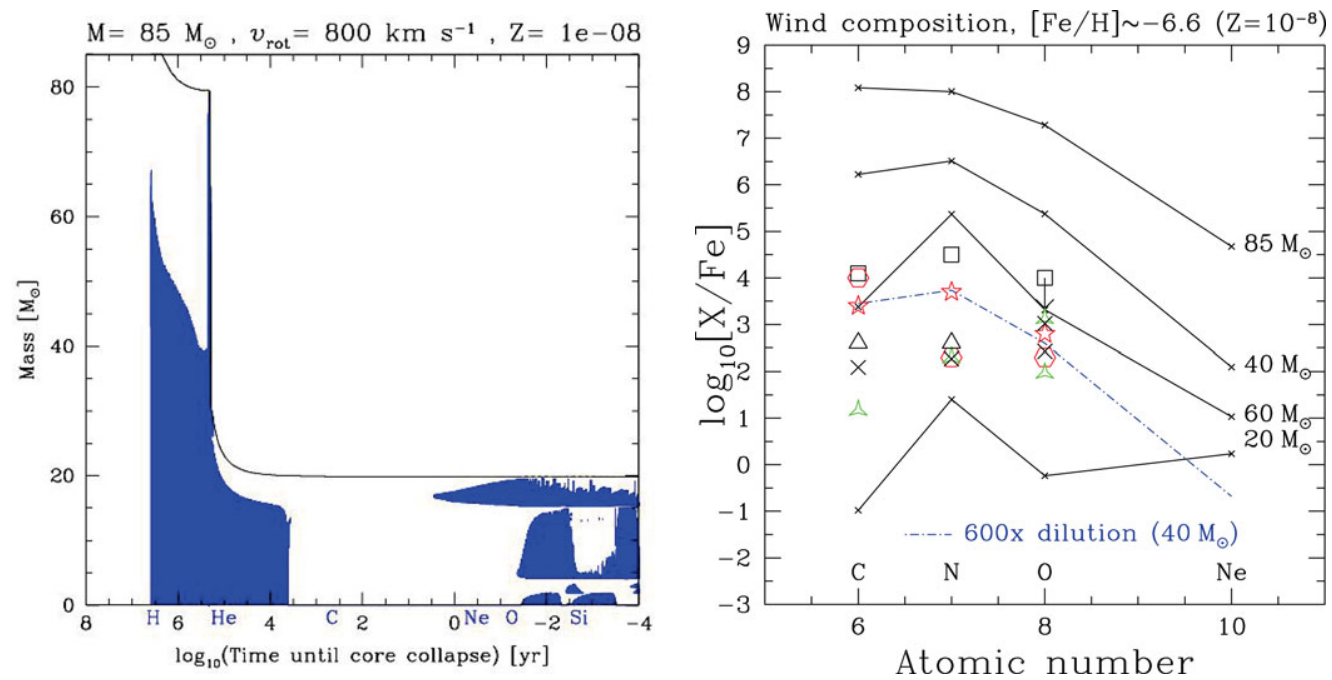

Figure 1. Left: Structure evolution diagram for a $85 M_{\odot}$ model with $v_{\text {ini }}=800 \mathrm{~km} \mathrm{~s}^{-1}$ at $Z=10^{-8}$. Coloured areas correspond to convective zones along the Lagrangian mass coordinate as a function of the time left until the core collapse. The burning stage abbreviations are given below the time axis. The top solid line shows the total mass of the star. Strong mass loss during the RSG stage removes a large fraction of total mass of the star. Right: Composition in $[\mathrm{X} / \mathrm{Fe}]$ of the stellar wind for the $Z=10^{-8}$ models (solid lines). For HE1327-2326 (red stars), the best fit for the CNO elements is obtained by diluting the composition of the wind of the $40 M_{\odot}$ model by a factor 600 (see Hirschi 2007 for more details).

- $M>60 M_{\odot}$ : high mass loss removes a significant amount of mass and the stars enter the WR phase. These stars therefore die as type Ib/c SNe and possibly as GRBs.

\section{Nucleosynthesis and galactic chemical evolution}

Rotation induced mixing leads to the production of primary nitrogen, ${ }^{13} \mathrm{C}$ and ${ }^{22} \mathrm{Ne}$. In this section, we compare the chemical composition of our models with carbon-rich EMP stars and include our stellar yields in a galactic chemical evolution (GCE) model and compare the GCE model with observations of EMP stars. We also study the weak s process production that can be expected with the primary ${ }^{22} \mathrm{Ne}$ obtained in our models.

\subsection{The most metal-poor star known to date, HE1327-2326}

Significant mass loss in very low- $Z$ massive stars offers an interesting explanation for the strong enrichment in CNO elements of the most metal-poor stars observed in the halo of the galaxy (see Meynet et al. 2006, Hirschi 2007). The most metal-poor star known to date, HE1327-2326 (Frebel et al. 2006) is characterised by very high N, C and O abundances, high $\mathrm{Na}, \mathrm{Mg}$ and $\mathrm{Al}$ abundances, a weak s-process enrichment and depleted lithium. The star is not evolved so it has not had time to bring self-produced CNO elements to its surface and is most likely a subgiant. By using one or a few SNe and using a very large mass cut, Limongi et al. (2003) and Iwamoto et al. (2005) are able to reproduce the abundances of most elements. However they are not able to reproduce the nitrogen surface abundance of HE1327-2326 without rotational mixing. The abundance pattern observed at the surface of that star presents many similarities with the abundance pattern obtained in the winds of very metal-poor fast rotating massive star models. HE13272326 may therefore have formed from gas, which was mainly enriched by stellar winds of rotating very low metallicity stars. In this scenario, a first generation of stars (PopIII) 


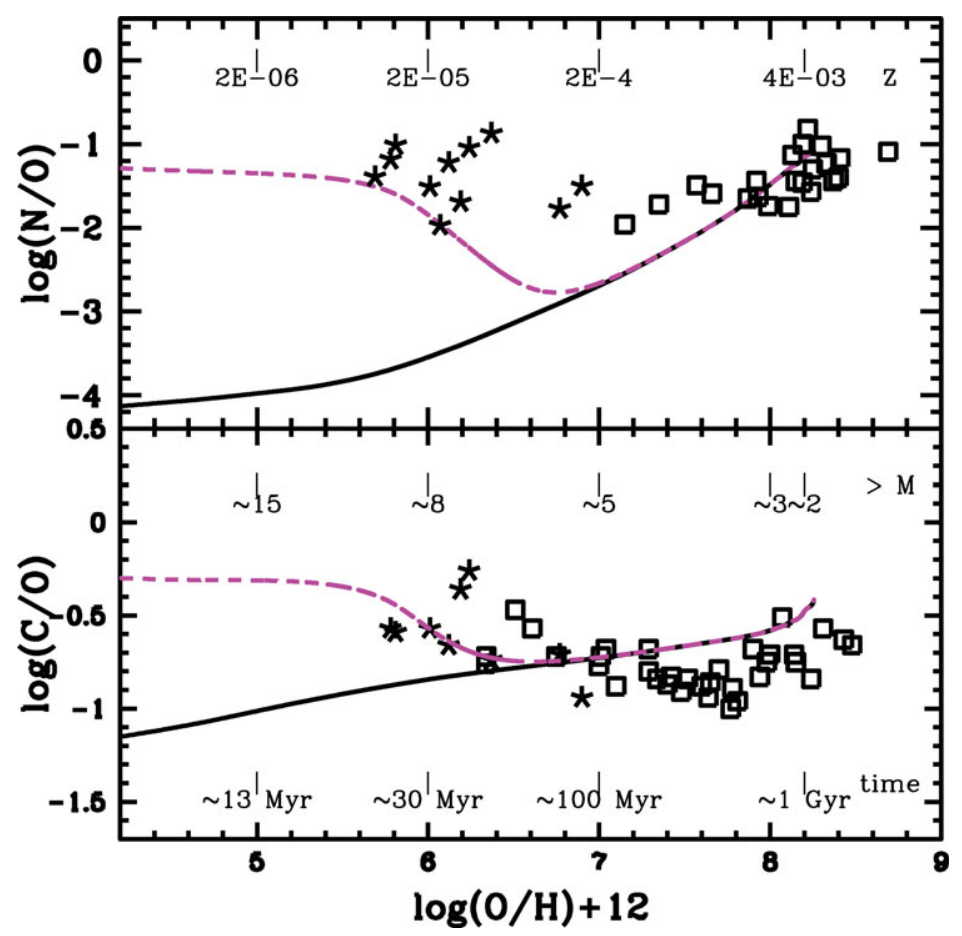

Figure 2. Chemical evolution model predictions of the N/O and $\mathrm{C} / \mathrm{O}$ evolution, in the galactic halo, for different stellar evolution inputs. The solid curves show the predictions of a model without fast rotators at low metallicities. The dashed lines show the effect of including a population of fast rotators at low metallicities (for the data see Chiappini et al. 2006a and references therein).

pollutes the interstellar medium to very low metallicities $([\mathrm{Fe} / \mathrm{H}] \sim-6)$. Then a PopII.5 star (Hirschi 2005), such as the $40 M_{\odot}$ model calculated here, pollutes (mainly through its wind) the interstellar medium out of which HE1327-2326 forms. This would mean that HE1327-2326 is a third generation star. The CNO abundances are well reproduced, in particular that of nitrogen, which according to Frebel et al. (2006) is 0.9 dex higher in [X/Fe] than oxygen. This is shown in Fig. 1 (right) where the abundances of HE1327-2326 are represented by the red stars and the best fit is obtained by diluting the composition of the wind of the $40 M_{\odot}$ model by a factor 600 . When the SN contribution is added, the $[\mathrm{X} / \mathrm{Fe}]$ ratio is usually lower for nitrogen than for oxygen. It is interesting to note that the very high $\mathrm{CNO}$ yields of the $40 M_{\odot}$ stars brings the total metallicity $Z$ above the limit for low mass star formation obtained in Bromm \& Loeb (2003).

\subsection{Primary nitrogen and ${ }^{13} \mathrm{C}$}

The high N/O plateau values observed at the surface of very metal poor halo stars require very efficient sources of primary nitrogen. Rotating massive stars can inject a large amount of primary $\mathrm{N}$ on short time scales. They are therefore very good candidates to explain the $\mathrm{N} / \mathrm{O}$ plateau observed at very low metallicity. According to the heuristic model of Chiappini et al. (2005), a primary nitrogen production of about $0.15 M_{\odot}$ per star is necessary. Using stellar yield calculations taking into account the effects of rotation at $\mathrm{Z}=10^{-8}$ in a chemical evolution model for the galactic halo with infall and outflow, both high $\mathrm{N} / \mathrm{O}$ and $\mathrm{C} / \mathrm{O}$ ratios are obtained in the very metal-poor metallicity range in agreement with observations (see details in Chiappini et al. 2006a). This model is shown 

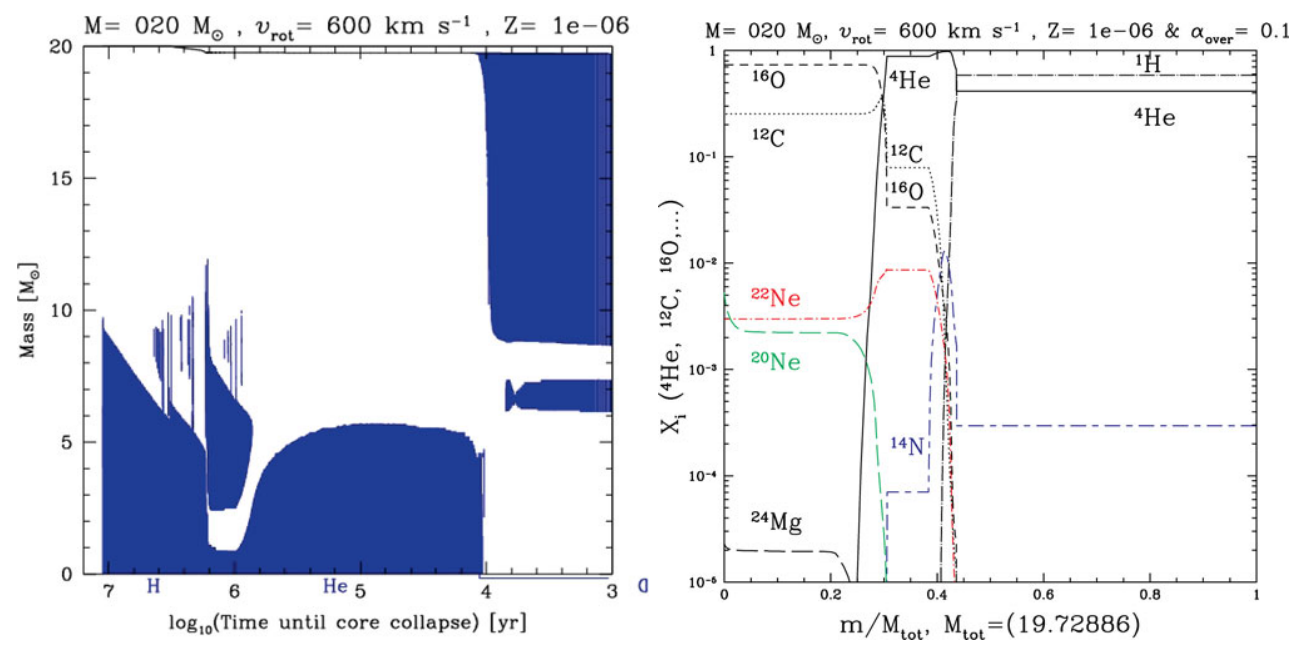

Figure 3. Left: Structure evolution diagram (see description of Fig. 1) for a rotating $20 M_{\odot}$ model at $Z=10^{-6}$ during $\mathrm{H}$ - and He-burning phases. Right: Chemical composition at the end of core He burning. Just above the core, one sees that the maximum abundance of ${ }^{22} \mathrm{Ne}$ is around $1 \%$ in mass fraction and at the end of core He burning, around $0.5 \%$ is burnt in the core, providing plenty of neutrons for s process.

in Fig. 2 (dashed magenta curve). In the same figure, a model computed without fast rotators (solid black curve) is also shown. Fast rotation enhances the nitrogen production by $\sim 3$ orders of magnitude. These results also offer a natural explanation for the large scatter observed in the N/O abundance ratio of normal metal-poor halo stars: given the strong dependency of the nitrogen yields on the rotational velocity of the star, we expect a scatter in the $\mathrm{N} / \mathrm{O}$ ratio which could be the consequence of the distribution of the stellar rotational velocities as a function of metallicity. As explained above, the strong production of primary nitrogen is linked to a very active H-burning shell and therefore a smaller helium core. As a consequence, less carbon is turned into oxygen, producing high $\mathrm{C} / \mathrm{O}$ ratios (see Fig. 2). Although the abundance data for $\mathrm{C} / \mathrm{O}$ is still very uncertain, a $\mathrm{C} / \mathrm{O}$ upturn at low metallicities is strongly suggested by observations (see Fabbian et al. 2008). Note that this upturn is now also observed in very metal poor DLA systems (Pettini et al. 2008).

In addition, stellar models of fast rotators have a great impact on the evolution of the ${ }^{12} \mathrm{C} /{ }^{13} \mathrm{C}$ ratio at very low metallicities (Chiappini et al. 2008). In this case, we predict that, if fast rotating massive stars were common phenomena in the early Universe, the primordial interstellar medium of galaxies with a star formation history similar to the one inferred for our galactic halo should have ${ }^{12} \mathrm{C} /{ }^{13} \mathrm{C}$ ratios between $30-300$. Without fast rotators, the predicted ${ }^{12} \mathrm{C} /{ }^{13} \mathrm{C}$ ratios would be $\sim 4500$ at $[\mathrm{Fe} / \mathrm{H}]=-3.5$, increasing to $\sim 31000$ at around $[\mathrm{Fe} / \mathrm{H}]=-5.0$ (see Fig.2 in Chiappini et al. 2008). Current data on EMP giant normal stars in the galactic halo (Spite et al. 2006) agree better with chemical evolution models including fast rotators. The expected difference in the ${ }^{12} \mathrm{C} /{ }^{13} \mathrm{C}$ ratios, after accounting for the effects of the first dredge-up, between our predictions with/without fast rotators is of the order of a factor of 2-3. However, larger differences (a factor of $\sim 60-90$ ) are expected for giants at $[\mathrm{Fe} / \mathrm{H}]=-5$ or turnoff stars already at $[\mathrm{Fe} / \mathrm{H}]=-3.5$. To test our predictions, challenging measurements of the ${ }^{12} \mathrm{C} /{ }^{13} \mathrm{C}$ in more extremely metal-poor giants and turnoff stars are required. 


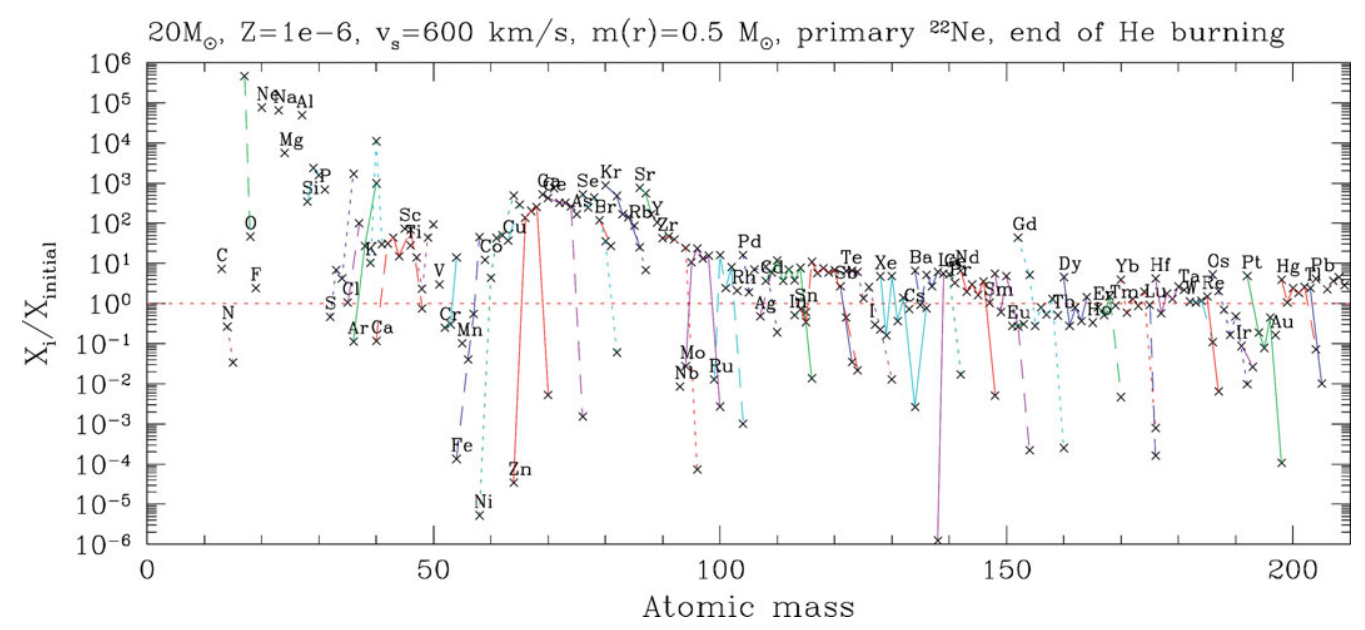

Figure 4. Left: Ratio of the abundance at the end of He-burning to the initial abundance for a model including primary ${ }^{22} \mathrm{Ne}$. We obtain large overproduction factors between 100 and 1000 in the mass range (A) 60 to 90 .

\subsection{Primary ${ }^{22} \mathrm{Ne}$ and $s$ process at low $Z$}

Models at $Z=10^{-8}$ show a production of primary ${ }^{22} \mathrm{Ne}$ during He burning. We also started calculating models at different $\mathrm{Z}$ to determine over which $\mathrm{Z}$ range the primary production of ${ }^{22} \mathrm{Ne}$ and also ${ }^{14} \mathrm{~N}$ is important. In Fig. 3, we show the properties of a 20 $M_{\odot}$ model at $Z=10^{-6}$ up to the end of He burning. Around $0.5 \%$ (in mass fraction) of ${ }^{22} \mathrm{Ne}$ is burnt during core He burning and therefore leads to a significant neutron release. We calculated the s process by using the primary nitrogen of the rotating $20 M_{\odot}$ model at $Z=10^{-6}$ inside a one-zone model (based on the Basel network and using an updated version of the reaclib reaction rate library) for s-process during He-burning. The first results are shown in Fig. 4. Large overproduction factors (100-1000) are obtained, however the process is not primary (see Pignatari et al. 2008 for more details).

\section{Conclusions and outlook}

The inclusion of the effects of rotation changes significantly the simple picture in which stellar evolution at low Z is just stellar evolution without mass loss. Strong mixing is induced between the helium and hydrogen burning layers leading to a significant production of primary ${ }^{14} \mathrm{~N},{ }^{13} \mathrm{C}$ and ${ }^{22} \mathrm{Ne}$. Rotating stellar models also predict strong mass loss during the RSG stage for stars more massive than $60 M_{\odot}$. These models predict the formation of $\mathrm{WR}$ and type $\mathrm{Ib} / \mathrm{c} \mathrm{SNe}$ down to almost $\mathrm{Z}=0$. The chemical composition of the stellar winds is compatible with the CNO abundance observed in the most metal-poor star known to date, HE1327-2326. GCE models including the stellar yields of these rotating star models are able to better reproduce the early evolution of $\mathrm{N} / \mathrm{O}, \mathrm{C} / \mathrm{O}$ and ${ }^{12} \mathrm{C} /{ }^{13} \mathrm{C}$ in our galaxy. These new stellar evolution models predict a large neutron release during core He burning and we present here the corresponding s-process production during $\mathrm{He}$ burning.

Large surveys of EMP stars (SEGUE, OZ surveys), of GRBs and SNe (Swift and GLAST satellites) and of massive stars (e.g. VLT FLAMES survey) are underway and will bring more information and constraints on the evolution of massive stars at low Z . On the theoretical side, more models are necessary to fully understand and study the 
complex interplay between rotation, magnetic fields, mass loss and binary interactions at different metallicities. Large grids of models at low $\mathrm{Z}$ will have many applications, for example to study the evolution of massive stars and their feedback in high redshift objects like Lyman-break galaxies and damped Ly-alpha systems.

\section{Acknowledgements}

R. Hirschi: Royal Society (2008/R1) and the organizers and M. Pignatari: MIRG-CT2006-046520 MC Grant (EU FP6), and the NSF grant PHY 02-16783 (JINA).

\section{References}

Asplund, M. 2005, ARAEBA, 43, 481

Beers, T. C. \& Christlieb, N. 2005, ARA\& $\mathcal{E}$, 43, 531

Bromm, V. \& Loeb, A. 2003, Nature, 425, 812

Chiappini, C., Ekström, S., Meynet, G., et al. 2008, A\&A, 479, L9

Chiappini, C., Hirschi, R., Matteucci, F., et al. 2006a, in Proceedings of Nuclei in the Cosmos IX, CERN, PoS(NIC-IX)080

Chiappini, C., Hirschi, R., Meynet, G., et al. 2006b, A\&AA, 449, L27

Chiappini, C., Matteucci, F., \& Ballero, S. K. 2005, A\& A, 437, 429

Chieffi, A. \& Limongi, M. 2004, ApJ, 608, 405

Ekström, S., Meynet, G., \& Maeder, A. 2007, ArXiv e-prints0709.0202, proc. "First Stars III"

Ekström, S., Meynet, G., Chiappini, C., et al. 2008, A\&A accepted, aph0807.0573

Fabbian, D., Nissen, P. E., Asplund, et al. 2008, in Conf. Precision Spectroscopy in Astrophysics, ed. Santos, N. C., et al., 45-46

Frebel, A., Christlieb, N., Norris, J. E., Aoki, W., \& Asplund, M. 2006, ApJ, 638, L17

Fukuda, I. 1982, PASP, 94, 271

Heger, A., Fryer, C. L., Woosley, S. E., Langer, N., \& Hartmann, D. H. 2003, ApJ, 591, 288

Heger, A. \& Langer, N. 2000, ApJ, 544, 1016

Heger, A. \& Woosley, S. E. 2002, ApJ, 567, 532

Hirschi, R. 2005, in IAU Symposium 228, ed. V. Hill, P. François, \& F. Primas, 331-332

Hirschi, R. 2007, A\& A, 461, 571

Hirschi, R., Chiappini, C., Meynet, G., et al. 2008, IAU250, 217-230, arXiv:0802.1675

Iwamoto, N., Umeda, H., Tominaga, N., Nomoto, K., \& Maeda, K. 2005, Science, 309, 451

Limongi, M., Chieffi, A., \& Bonifacio, P. 2003, ApJ, 594, L123

Maeder, A., Grebel, E. K., \& Mermilliod, J.-C. 1999, A\& $A$, 346, 459

Maeder, A. \& Meynet, G. 2005, A\&A, 440, 1041

Marigo, P. 2002, $A \mathscr{E} A, 387,507$

Martayan, C., Floquet, M., Hubert, A. M., et al. 2007, A\& A, 472, 577

Meynet, G., Ekström, S., \& Maeder, A. 2006, A\&A, 447, 623

Meynet, G., Ekström, S., \& Maeder, A., et al. 2008, IAU250, 147-160, arXiv:0802.2805

Meynet, G. \& Maeder, A. 2000, $A \& A, 361,101$

-. 2002, A\&A, 390, 561

-. 2005, A\&A, 429, 581

Pettini, M., Zych, B. J., Steidel, C. C., et al. 2008, MNRAS, 385, 2011

Pignatari, M., Gallino, R., Meynet, G., et al. 2008, ApJL accepted

Pustilnik, S. A., Tepliakova, A. L., Kniazev, A. Y., et al. 2008, MNRAS, 388, L24

Schneider, R., Omukai, K., Inoue, A. K., \& Ferrara, A. 2006, MNRAS, 369, 1437

Spite, M., Cayrel, R., Hill, V., et al. 2006, A\&A, 455, 291

Spite, M., Cayrel, R., Plez, B., et al. 2005, A\& $A$, 430, 655

Spruit, H. C. 2002, A\&AA, 381, 923

Tominaga, N., Umeda, H., \& Nomoto, K. 2007, ApJ, 660, 516 